\title{
Nonlinear Polarization Rotation Characteristic Phenomenon in a Bulk Semiconductor Optical Amplifier
}

\author{
Xanghua Feng ${ }^{1}$, Jiarong $\mathrm{Ji}^{2}$, Guomin Zhang ${ }^{1}$ \\ ${ }^{1}$ College of Science,Information Engineering University, Zhengzhou, China \\ ${ }^{2}$ College of Optoelectronic Science and Engineering National University of Defense Technology, Changsha, China \\ Email: Fengxianghua2002@hotmail.com
}

Received 2013

\begin{abstract}
The phenomena of polarization rotation induced by self-modulation in semiconductor optical amplifier (SOA) are analyzed theoretically. The relationship between polarization parameters and gain as well as phase is obtained by the correlation parameter of ellipse polarization and SOA nonlinearity polarization rotation theory. The experiment employs polarizer drive by walking electromotor and power meter, the light power of 360 degree is measured. The transformation law of output polarization power components is found for obvious polarization rotation in the selected coordinate axes based on connection of polarization state in difference axes. Using this law make the manipulation easily on getting ideal polarization state. It can offer a fine method to realize all-optical switch and other logic elements in experiment. This work is of great significance for the applications of SOA nonlinear polarization rotation at high-speed all-optical signal processing and all-optical logic gate.
\end{abstract}

Keywords: Strained Bulk SOA; Nonlinear Polarization Rotation; Phase Difference; Polarization Azimuth

\section{Introduction}

H.Soto and D.Erasme [1] first raised a concept of crosspolarization-modulation (XPolM) in 1999. They investigate the transformation law of SOA birefraction with Jone-matrix. The nonlinear polarization rotation induced by SOA birefraction was used on all-optical wavelength conversion, switch, logic gate in the paper [2,3].

From 2003, Dorren $[4,5]$ et al. researched all-logic elements and all-optical switch base on XPolM in theory and experiment, and raised a theory model for polarization dependent gain saturation in tensile strain bulk SOA. L.Q.Guo et al. set a $2.5 \mathrm{Gbit} / \mathrm{s}$ all-optical AND gate base on XPolM [6-8]. They proved extinction ratio increased 8dB contrast all-optical AND gate base on cross-gainmodulation. Due to the polarization rotation can induce the variety of output power, the polarization rotation combining other non-linearity effect can use in all-optical nonlinear regeneration [9] and all-optical sampling [10] and optical buffer [11] and optical data comparator [12]. Recently, with investigate deeply, the applications of SOA nonlinear polarization rotation at high-speed all-optical signal processing and all-optical logic are more and more widespread along with research deeply $[13,14]$.

\section{Theory Model and Simulations}

The tensile strain bulk SOA is used for experiment. Based on Dorren's theory, The polarized optical field are decomposed into a transverse electric (TE) and trans- verse magnetic (TM) component. TE and TM mode propagate "independently" through the SOA, although they have indirect interaction with each other via the gain saturation. Because of different confinement factor and effective refractive indexes experienced by TE and TM modes, it results in a phase shift TE and TM modes of the light upon leaving the SOA. When a light transit SOA, the out powers for each polarization can be expressed as:

$$
\begin{gathered}
P_{\text {out }}^{T E}(\tau)=P_{\text {in }}^{T E}(\tau) \exp \left(\frac{\Gamma_{T E} g_{T E} L}{v_{g}}-\alpha_{\mathrm{int}}^{T E}\right) \\
P_{\text {out }}^{T M}(\tau)=P_{\text {in }}^{T M}(\tau) \exp \left(\frac{\Gamma_{T M} g_{T M} L}{v_{g}}-\alpha_{\mathrm{int}}^{T M}\right)
\end{gathered}
$$

where $P_{\text {in }}$ is input power, $P_{\text {out }}$ is output power, $\Gamma$ is the confinement factor, $g$ is the gain, $L$ is the length of the SOA.

By relation of gain and phase, output phase through $L$ can be expressed as

$$
\begin{aligned}
& \phi_{T E}=\phi_{T E 0}-\frac{L}{2} \frac{\Gamma_{T E} g_{T E} \alpha_{T E}}{v_{g}} \\
& \phi_{T M}=\phi_{T M 0}-\frac{L}{2} \frac{\Gamma_{T M} g_{T M} \alpha_{T M}}{v_{g}}
\end{aligned}
$$

where $\alpha$ is phase modulation coefficients, it express the 
coupling relation of gain and refractive index in SOA.

Through $L$, the phase difference between TE and TM modes can be computed from (3) and (4)

$$
\Delta \phi=\phi_{T E}-\phi_{T M}
$$

In the most simple approach, one would choose $\alpha_{T M}=\alpha_{T E}$. By(1)-(5), we can get:

$$
\Delta \phi=\frac{\alpha_{T E}}{2}\left(\ln \frac{P_{\text {out }}^{T M}}{P_{\text {out }}^{T E}}-\ln \frac{P_{\text {in }}^{T M}}{P_{\text {in }}^{T E}}\right)+\Delta \phi_{0}
$$

(6) express the relation of phase difference and input/ output power. Due to the change of TE and TM output powers, the phase difference between TE and TM modes change. $\ln \frac{P_{\text {out }}^{T M}}{P_{\text {out }}^{T E}}$ is more and the phase difference is more, as Figure 1. Three curves represent the phase difference range of $80^{\circ}$ (curve1), $100^{\circ}$ (curve3) and $120^{\circ}$ (curve2). The calculation parameters of this paper use parameters of literature [4].

It is get different rotation degree by same input phase difference and different TE/TM input powers. It is also get different rotation degree by different input phase difference and same TE/TM input powers. As Figure 2, the rotation degree of curve1 is more. More rotation degree can be obtained by chose right input phase difference.

Definition extinction ratio $c=10 \log \left(\frac{P_{\text {long axis }}}{P_{\text {short axis }}}\right) \cdot$ The output extinction ratio change with input polarization state. The linear output can be obtained by adjusting input phase difference at arbitrarily input powers, as Figure 3. Curve 1 express when input power is $-1 \mathrm{dBm}$, Curve 2 express when input power is $-5.9 \mathrm{dBm}$, output light is linearly polarized light.

The experiment employs polarizer driving by walking electromotor and power meter. If the polarizer axis $(x, y$ axis) is different to SOA perpendicular axis (TE, TM

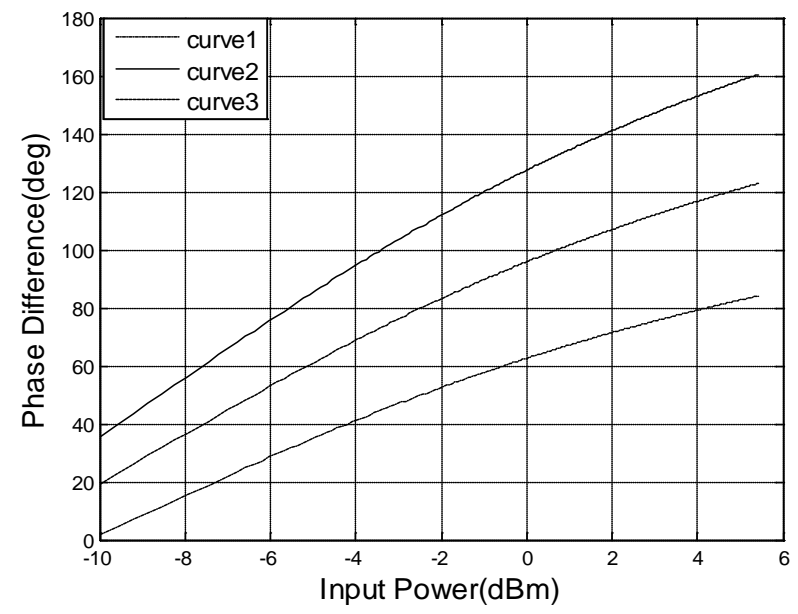

Figure 1. Phase difference variations depend on different TE mode and the TM mode output power.

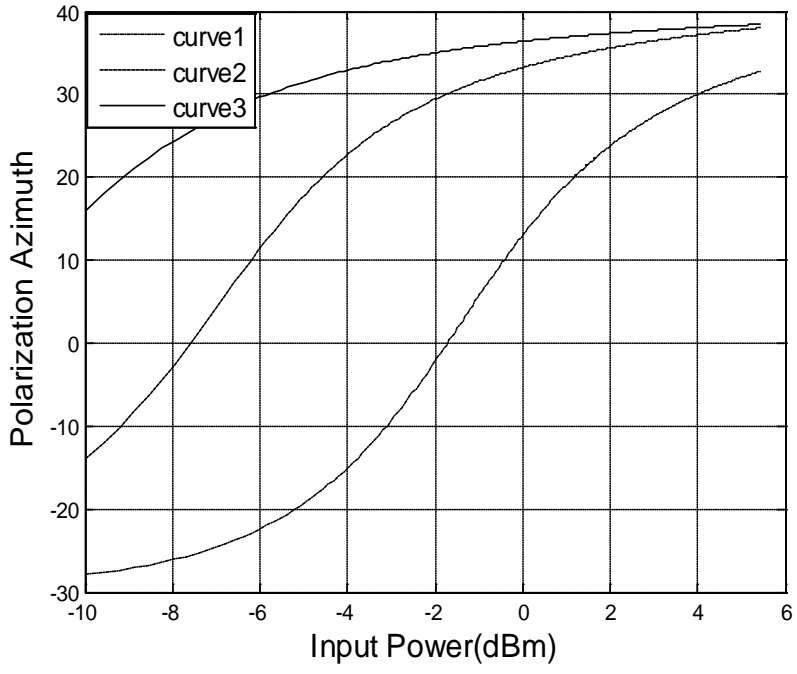

Figure 2. Polarization Azimuth variations depend on different phase difference of TE mode and the TM mode.

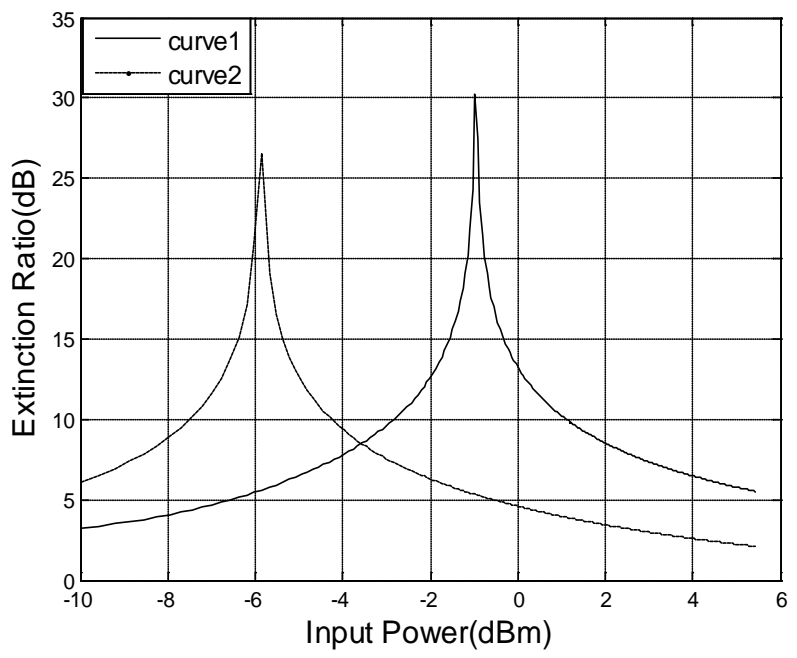

Figure 3. Extinction ratio variations depend on different phase difference of TE mode and the TM mode

axis), the power satisfy:

$$
\begin{gathered}
P_{x}=P_{T E} \cos ^{2} \varphi+P_{T M} \sin ^{2} \varphi-\sqrt{P_{T E} P_{T M}} \sin 2 \varphi \cos \Delta \phi \\
P_{y}=P_{T E} \sin ^{2} \varphi+P_{T M} \cos ^{2} \varphi+\sqrt{P_{T E} P_{T M}} \sin 2 \varphi \cos \Delta \phi
\end{gathered}
$$

where $\varphi$ is angle between $x$ axis and TE axis. $P_{x}$ and $P_{y}$ are affected by $\Delta \phi$ obviously. The phase difference cosine law appear on $x y$ axis from Figure 4. We can speculate cosine changing is more obvious, polarization state changing is more. The biggest difference between $P_{x}$ and $P_{y}$ is more, output light is more close to linearly polarized light. It means SOA output polarization state variety with input power is known by polarizer. So experiment operation become easily. So long as adjusting suitable input polarization state, we can get expect polarization state at arbitrarily input power. 


\section{Experimental Results}

Experimental setup is used to measure SOA nonlinear polarization rotation as Figure 5. A commercial polarization-independent SOA is employed. Its saturation power is $10 \mathrm{dBm}$. A walking electromotor controlled by computer drive a polarizer, so the light power of every orienttation is measured. The walking electromotor rotate circuit, sampling point is 400 . The distribution of power is displayed on computer.

In this paper, two input pump polarization states(A and B) are selected as contrast. Figure 6 is an input and output polarization light power of A polarization state corresponding with 360 degree.

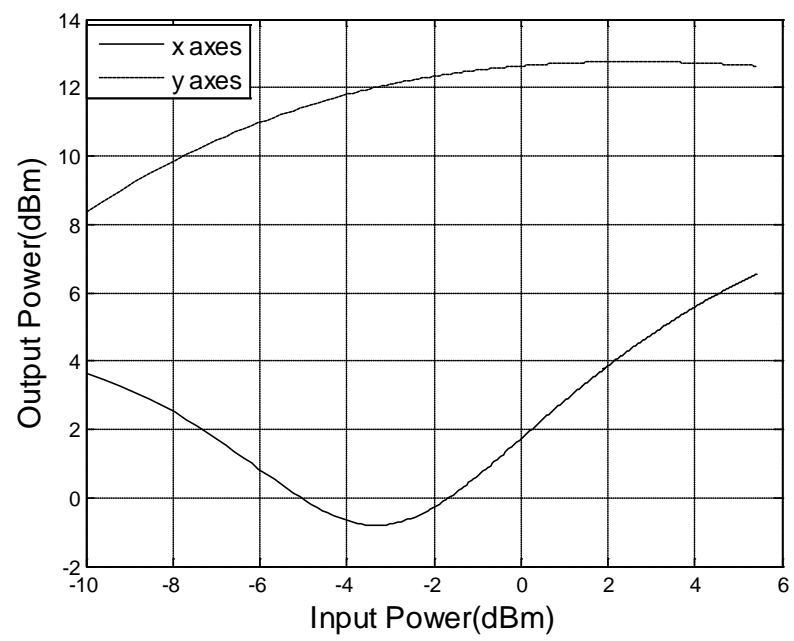

Figure 4. SOA output power on $x y$ perpendicular axes.

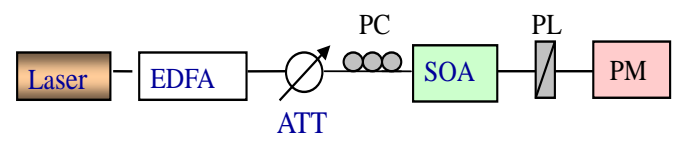

Figure 5. Experimental setup is used to measure SOA polarization power. EDFA: erbium-doped fiber amplifier, ATT: Attenuator, PC: Polarization controller, PL: Polarizer, PM: Power meter.

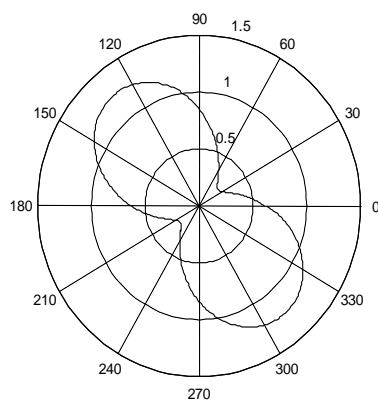

(a)

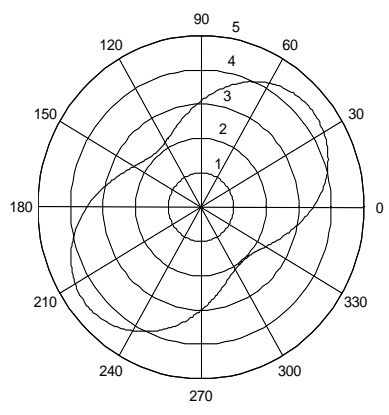

(b)
Figure 6. Input and output polarization light power of A polarization state correspond with 360 degree. (a) Input; (b) output.
Figure 7 is an input and output polarization light power of A polarization state corresponding with 360 degree.

In Figures 6 or 7, long axis power $P_{\xi}$, short axis power $P$, angle $\theta$ of $x$ axis and long axis, $x$ axis power $P_{x}, y$ axis power $P_{y}, 45^{0}$ power $P_{45}$ are easy to find out. According to transform relation of different axis,

$$
P_{45}=P_{x} \cos ^{2} \frac{\pi}{4}+P_{y} \sin ^{2} \frac{\pi}{4}-\sqrt{P_{x} P_{y}} \sin 2 \frac{\pi}{2} \cos \Delta \phi
$$

get:

$$
\cos \Delta \phi=\frac{\left(P_{x}+P_{y}\right) / 2-P_{45}}{\sqrt{P_{x} P_{y}}}
$$

For arbitrary orthogonal axis, phase difference between perpendicular components can be getting at this coordinate.

Figure 8 is output probe power $P_{x}, P_{y}$ corresponding $\mathrm{A}$ and $\mathrm{B}$.

Figure 9 is polarization Azimuth variation curves correspond to $\mathrm{A}$ and $\mathrm{B}$. Because polarization state drift and systemic vibration make power measurement error, polarization azimuth extinction ratio will produce error. Figure 10 is extinction ratio variation curves correspond to $\mathrm{A}$ and B. Figure 11 is phase difference of $x$ and $y$ variation curves correspond to $\mathrm{A}$ and $\mathrm{B}$.

\section{Conclusions}

From ahead experimental result, we can get the conclusion. Polarization state rotation always occurs through SOA, rotation degree is different follow different input power and input polarization state. Two different input polarization states lead to output power and gain obvious difference of $x$ and $y$ axis. Their phase difference, polarization Azimuth and extinction ratio are difference obviously. Due to $x$ and $y$ axis do not coincide TE and TM axis, obvious cosine variation curve is observed at Figure 8(a). It implies input polarization state of Figure 6(a) arise phase difference of TE and TM modes bigger change, and it arise rotation bigger degree and extinction ratio

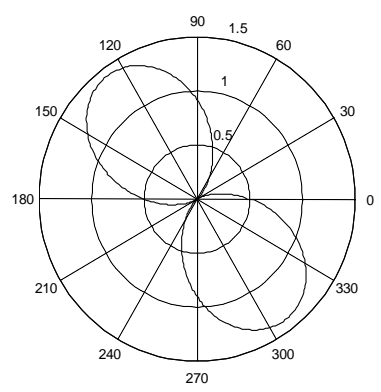

(a)

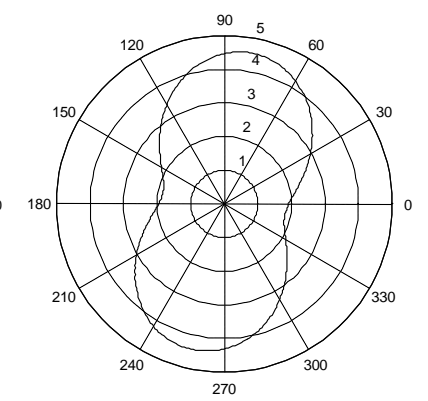

(b)
Figure 7. Input and output polarization light power of $B$ polarization state correspond with 360 degree. (a) Input; (b) output 


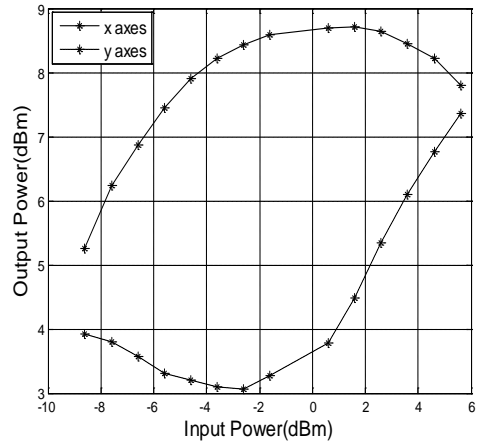

(a)

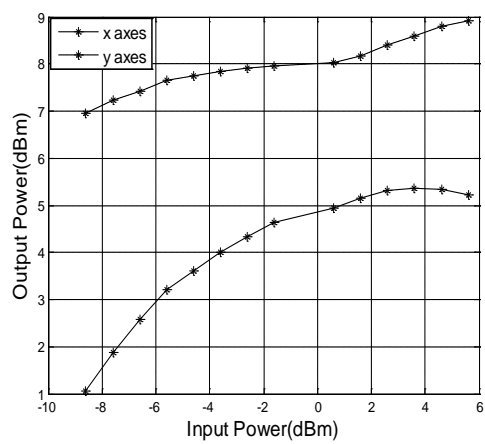

(b)

Figure 8. Output polarization light power. (a) Correspond to A polarization state; (b) correspond to $B$ polarization state.

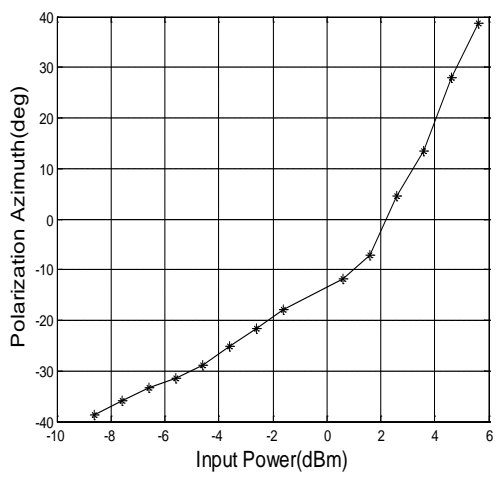

(a)

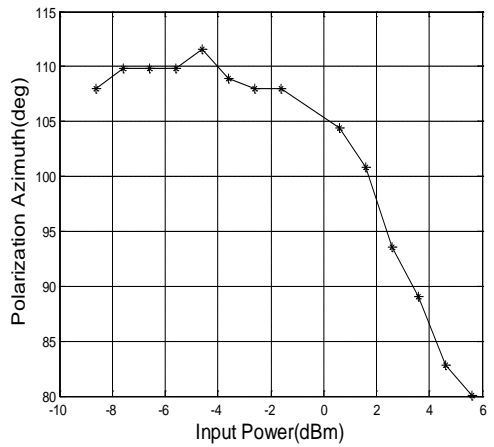

(b)

Figure 9. Polarization azimuth variation curve. (a) Correspond to A polarization state; (b) correspond to B polarization state.

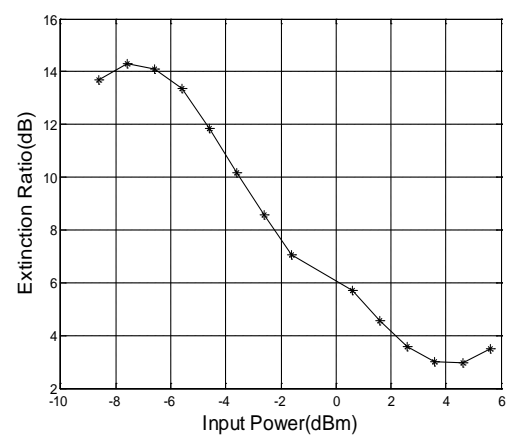

(a)

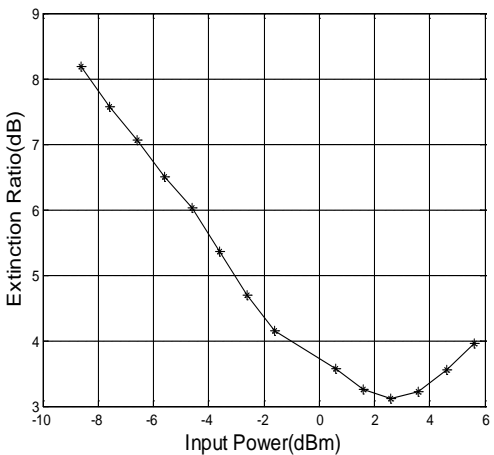

(b)

Figure 10. Extinction ratio variation curve. (a) Correspond to A polarization state; (b) correspond to $B$ polarization state.

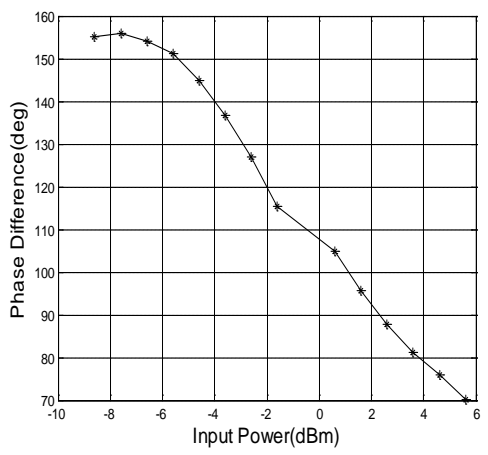

(a)

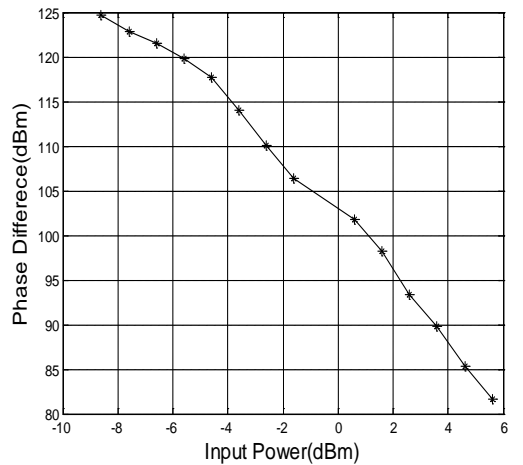

(b)

Figure 11. Phase difference variation curve. (a) Correspond to A polarization state; (b) correspond to $B$ polarization state. 
bigger change. Cosine variation curve is not observed at Figure 8(b). It implies input polarization state of Figure 7(a) do not arise phase difference of TE and TM modes bigger change, and it not arise rotation and extinction ratio bigger change. Thus it can be seen, if input polarization state arise phase difference of TE and TM modes bigger change, output powers at $x$ axis or $y$ axis appear cosine variation. It proves in theory and experiment, arbitrary output probe polarization states can be obtained through adjusting input pump polarization states. In experiment, the adjusting of polarization states can manipulate easily base on transform relation of different coordinate system.

\section{REFERENCES}

[1] H.Soto, D. Erasme and G. C. Guekos, "Cross-Polarization Modulation in Semiconductor Optical Amplifiers,” IEEE Photonics Technology Letters, vol. 11, No. 8, 1999, pp. 970-972.doi:10.1109/68.775316

[2] H. Soto, D. Erasme, G. C. Guekos, et a1., "5-Gb/s XOR Optical Gate Based on Cross-Polarization Modulation in Semiconductor Optical Amplifiers," IEEE Photonics Technology Letters, Vol. 13, No. 4, 2001, pp. 335-337. doi:10.1109/68.917843

[3] H. Soto, C. A. Diaz, J. Topomondzo, et a1. "All-Optical AND Gate Implementation Using Cross-Polarization Modulation in A Semiconductor Optical Amplifier," IEEE Photonic Technology Letters, Vol. 14, No. 4, 2002, pp. 498-500. doi:10.1109/68.992590

[4] H. J. S. Dorren, D. Lenstra, Y. Liu, "Nonlinear Polarization Rotation in Semiconductor Optical Amplifiers: Theory and Application to All-Optical Flip-Flop Memories,” IEEE Journal of Quantum Electronics, Vol. 39, No. 1, 2003, pp. 141-148. doi:10.1109/JQE.2002.806200

[5] H. J. S. Dorren, X. L. Yang and A. K. Mishra, “A11Optical Logic Based on Ultrafast Gain and Index Dynamics in a Semiconductor Optical Amplifier," IEEE Journal of Selected Topics in Quantum Electronics, Vol. 10, No. 5, 2004, pp. 1079-1092. doi:10.1109/JSTQE.2004.835293(410)1

[6] L. Q. Guo and M. J. Connelly, “All-Optical AND Gate with Improved Extinction Ratio Using Signal Induced Nonlinearities in A Bulk Semiconductor Optical Amplifier," Optical Express, Vol. 14, No. 7, 2006, pp. 2938-2943.

\section{doi:10.1364/OE.14.002938}

[7] L. Q. Guo and M. J. Connelly, “A Mueller-Matrix Formalism for Modeling Polarization Azimuth and Ellipticity Angle in Semiconductor Optical Amplifiers in a Pump-Probe Scheme,” Journal of Lightwave Technology, Vol. 25, No. 1, 2007, pp. 410-420.

doi:10.1109/JLT.2006.886674

[8] L. Q. Guo and M. J. Connelly, “A Poincare Approach to Investigate Nonlinear Polarization Rotation in Semiconductor Optical Amplifiers and Its Application to All-Optical Wavelength Conversion," Proceedings of SPIE, Vol. 6783, No. 678325, 2007, pp. 1-5. doi:10.1117/12.745453

[9] P. Morin, J. Fatome, C. Finot, et al., "All-Optical Nonlinear Processing of Both Polarization State and Intensity Profile for 40 Gbit/s Regeneration Applications," Optics Express, Vol. 19, No. 18, 2011, pp. 17158-17166. doi:10.1364/OE.19.017158

[10] S. Zhang, Y. Liu, et al., “All-Optical Sampling Based on Nonlinear Polarization Rotation in Semiconductor Optical Amplifiers,” Journal of Optoelectronics and Biomedical Materials, Vol. 1, No. 4, 2009, pp. 383-388.

[11] M. Cheng, C. Q. Wu, et al., "Cascaded Optical Buffer Based on Nonlinear Polarization Rotation in Semiconductor Optical Amplifiers," Chinese. Physics. Letters, Vol. 25, No. 11, 2008, pp. 4026-4629. doi:10.1088/0256-307X/25/11/052

[12] S. K. Garai, “A Method of Developing Frequency Encoded Multi-Bit Optical Data Comparator Using Semiconductor Optical Amplifier,” Optics \& Laser Technology, Vol. 43, No. 1, 2011, pp. 124-131. doi:10.1016/j.optlastec.2010.05.016

[13] L. L. Yi, W.S. Hu, et al., “All-Optical Reconfigurable Multi-Logic Gates Based on Nonlinear Polarization Rotation Effect in A Single SOA," Chinese Optics Letters, Vol. 9, No. 3, 2011, pp.030603(1-4).

[14] Z. Y. Li, C. Q. Wu, H. Dong, et a1.,"Matrix Analysis for Polarization Rotation of Semiconductor Optical Amplifiers with Related Application,” Chinese J. Lasers, Vol. 36, No. 4, 2009, pp. 842-845. doi:10.3788/CJL20093604.0842

[15] S. K. Garai, P. Ghosh and S. Mukhopadhyay, "Analytical Approach of Developing Wavelength Encoded AND, NAND and X-OR Logic Operations and Implementation of the Theory Using Semiconductor Optical Amplifiers," Optik, Vol. 122, No. 7, 2011, pp. 569-576. doi:10.1016/j.ijleo.2010.04.012 